\title{
Die Mathematische Modellsammlung der Philipps-Universität Marburg
}

\author{
Ilka Agricola
}

Der Ars legendi-Fakultätenpreis in der Kategorie Mathematik ging in diesem Jahr an Ilka Agricola von der PhilippsUniversität Marburg. Die Preisverleihung fand am 5. April 2016 im ScienceCenter Experiminta in Frankfurt am Main statt. In dem folgenden Beitrag schildert die Preisträgerin die Geschichte der Mathematischen Modellsammlung der Philipps-Universität Marburg und ihre Verwendung in der Lehre.

\section{Die Anfänge der Sammlung}

Es waren einmal ... ein paar verstaubte Kisten in der Teeküche, die traurigen Überreste einer Sammlung, die um 1885, in etwa zeitgleich mit der Gründung des Fachbereichs (damals noch Mathematisches Seminar), angelegt wurde.'

Heinrich Weber (I842-1913) war von 1884 bis 1892 Professor für Mathematik an der Universität Marburg und gilt als einer der aktivsten Mathematiker seiner Zeit; seine Forschungen reichten von der Algebra (Begriff des Normalteilers, Satz von Kronecker-Weber) über die Funktionentheorie bis zur Analysis (Einführung der Weber-Funktionen, heute bekannt als Bessel-Funktionen 2. Art). Er hatte am II. November 1884 die Gründung eines mathematischen Seminars und Mittel zu dessen Einrichtung beantragt, beides wurde am 23. Januar 1885 bewilligt. Die gesamte Universität hatte damals um die 1000 Studenten, die Mathematik zwischen 10 und 30 Studen-

ten. Bis zum Sommersemester 1904 stiegt die Zahl dann langsam auf 75 Studenten an.

Nach der Gründung des mathematischen Seminars begann man, eine Sammlung von Modellen zur Unterstützung der Lehre anzulegen. Aus dieser Zeit sind noch einige Gips- und Kartonmodelle von Flächen sowie Vermessungsinstrumente und Planimeter erhalten. Bis etwa 1960 wurde die Sammlung entsprechend den Schwerpunkten der Professoren in Lehre und Forschung kontinuierlich erweitert. Unterlagen zur Sammlung finden sich in den Archiven leider nur selten, selbst Rechnungen sind oft nicht erhalten geblieben. Eine Ausnahme stellt ein Brief vom 6. Juli 1932 dar, in dem der damalige Vertreter der angewandten Mathematik, Prof. Maximilian Krafft, die schlechte Ausstattung beklagte und einen Zuschuss von 535 Mark zur Anschaffung und Instandsetzung einiger gebrauchter Geräte beantragte. So kam der Fachbereich in den Besitz eines Pantografen, Baujahr 1866, und eines Theodoliten, Baujahr 1906, der Firma Breithaupt in Kassel, die auch heute noch als Hersteller von Präzisionsmessinstrumenten bekannt ist. Beide Instrumente sind voll funktionsfähig und heute in der Bibliothek des Fachbereichs ausgestellt.

Der durch Nicolas Bourbaki ausgelöste Abstraktionsschub nach dem 2. Weltkrieg war für die Entwicklung der modernen Mathematik essenziell, führte aber leider auch dazu, dass Modelle als antiquiert galten und fortan verpönt waren. Die Marburger Sammlung wurde auseinandergerissen und geriet in Vergessenheit.

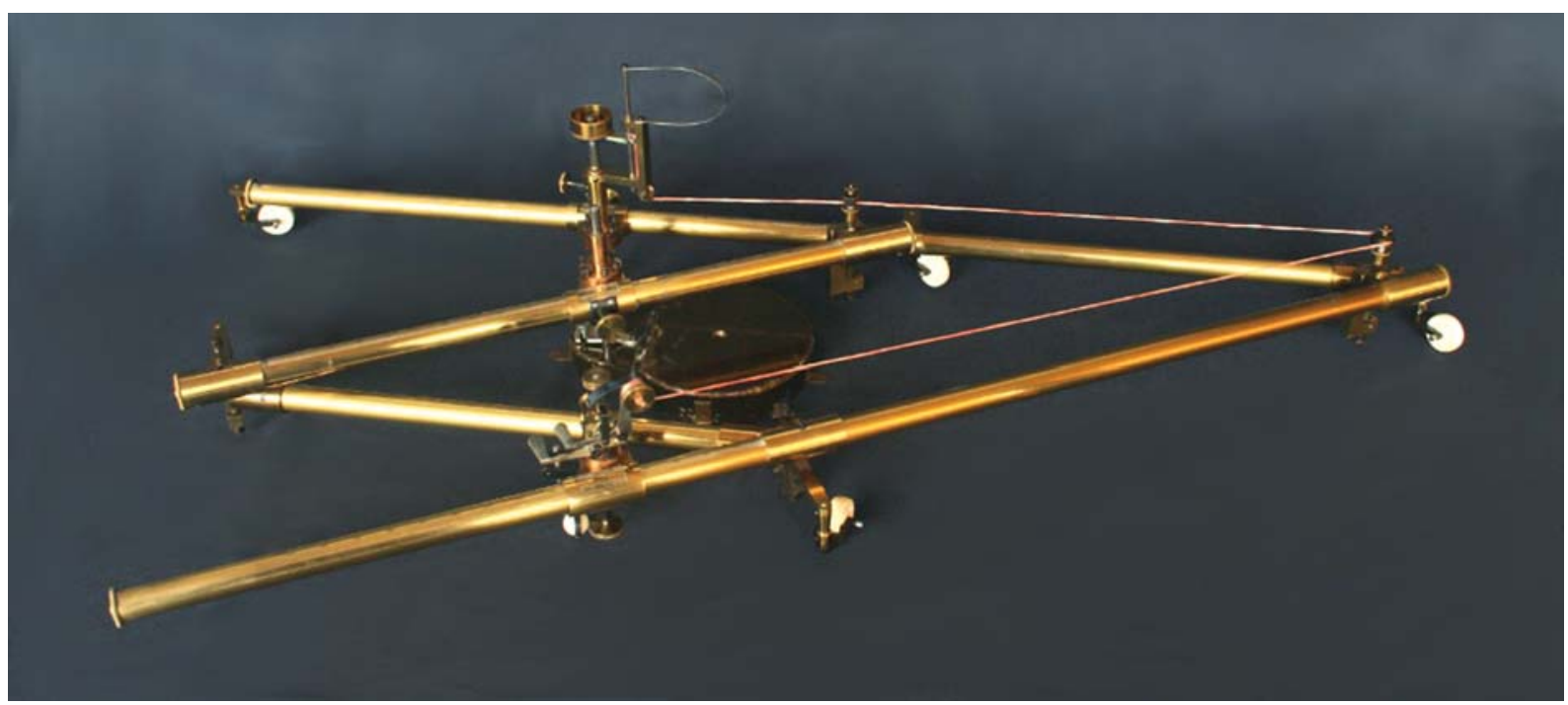

Breithaupt-Pantograf aus Messing (Foto: Ramona Trusheim) 


\section{Der Neuanfang}

Nach Jahrzehnten des Verfalls gab es irgendwann nur noch zwei Alternativen: neu anfangen oder wegwerfen. Zum Glück entschied man sich für die erste Variante und so wurde im Herbst 2008 damit begonnen, das Vorhandene zu sammeln, zu reparieren, zu ergänzen und zu inventarisieren. Die dafür notwendigen Mittel stellte das Präsidium zur Verfügung, mit zusätzlicher Unterstützung durch den Universitätsbund. Die empfindlichsten Stücke wurden von einem professionellen Restaurator bearbeitet: Joachim Jendersie aus Jena, der hier Hervorragendes geleistet hat. Für alle anderen Exponate mussten wir improvisieren, Handwerker oder andere Sammlungen um Rat fragen - wie repariert man etwa die klasischen Gipsflächen von Alexander Brill aus dem 19. Jahrhundert?

Die meisten Vitrinen konnte die Sammlung aus Museen, anderen Sammlungen oder Fachbereichen, der Universitätsbibliothek usw. gebraucht übernehmen. Das klingt einfacher und preiswerter, als es ist: Auf- und Abbau, Transport, Ausbesserung von Schadstellen, Neuanfertigung von Fachböden, Ersatz defekter Schlösser und fehlender Schlüssel, Anschluss der Vitrinenbeleuchtung (wo vorhanden) ans Stromnetz ... Um am Ende fertige Vitrinen am richtigen Standort zu haben, ist die Unterstützung von Fahrern, Hausmeistern, Elektrikern, Feinmechanikern, Schreinern, Glasern und vielen starken freiwilligen Helfern notwendig; Museumsglas ist schwer. Nie wieder werde ich in einem Museum leichtfertig an einer großen Vitrine vorbei schlendern.

Zum Festkolloquium anlässlich des 125-jährigen Bestehens des Fachbereichs am 2. Februar 201I wurde die Sammlung offiziell wiedereröffnet.

Unter dem Namen Mathematische Modellsammlung wird heute die gesamte Sammlung des Fachbereichs Mathematik und Informatik verstanden, auch wenn sie inhaltlich deutlich über rein mathematische Modelle hinausgeht und auch Leihgaben anderer Fachbereiche und des Hochschulrechenzentrums umfasst. Im Foyer und in der Bibliothek sind in einem guten Dutzend Vitrinen die schönsten Stücke ausgestellt; zwei Räume dienen der Sammlung als Werkstatt, Archiv, Lagerraum und Arbeitsplatz gleichermaßen. Insbesondere lagern dort Objekte, die nur in der Lehre verwendet werden oder für die es noch keine geeigneten Vitrinen gibt.

Was für eine Sammlung soll es sein?

In Zeiten knapper Kassen stellt sich für jede wissenschaftliche Sammlung die Frage, welche Funktion sie erfüllen soll, da doch die die Hauptaufgaben der Universitäten Forschung und Lehre sind, nicht der Bau und Unterhalt von Museen. Offensichtlich hängt von der Beantwortung

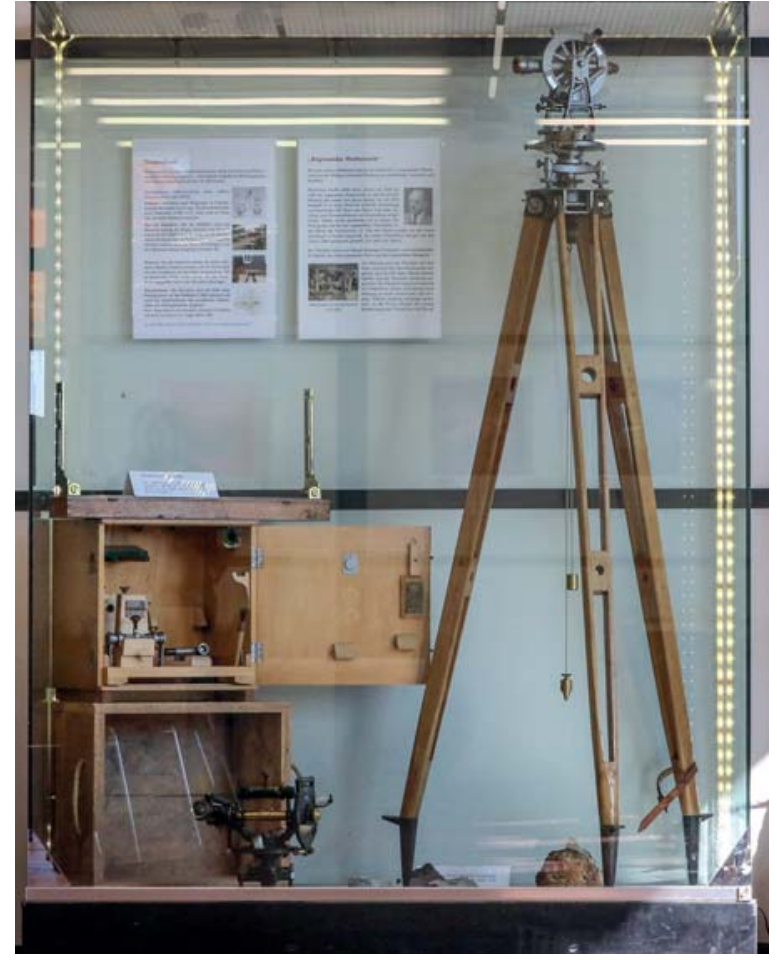

Eine der Vitrinen in der Bibliothek (Foto: Thorsten Richter)

dieser Frage ganz entscheidend ab, wie mit einer Sammlung gearbeitet wird und wie man sie weiterentwickelt. Die wiedergegründete Mathematische Modellsammlung in Marburg hat ihrem Selbstverständnis nach in erster Linie keinen musealen Charakter - auch wenn sie über einige wertvolle „Museumsstücke“ verfügt -, sondern ist eine Lehrsammlung für die Ausbildung von Mathematikern und Informatikern in den Bachelor- und Lehramtsstudiengängen.

Eine erste überraschende Erfahrung mit den Studierenden, aber auch mit Kollegen aus dem In- und Ausland, ist, dass viele mathematische Geräte der Vergangenheit auch für einen aktiven Wissenschaftler kaum mehr selbsterklärend, meist sogar völlig unbekannt sind: Kaum jemand kennt noch ein Planimeter (zur Ausmessung von Flächeninhalten, etwa auf einer Landkarte) oder einen harmonischen Analysator (zur Berechnung der FourierKoeffizienten einer periodischen Funktion, z. B. zur Zerlegung eines akustischen Signals in seine Obertöne oder zur Gezeitenvorhersage); auch Rechenschieber sind seit ihrer Ersetzung durch den Taschenrechner in den 1970er Jahren kaum mehr bekannt. Verständliche Erläuterungen und eine sinnvolle Anordnung der Exponate sind deswegen essenziell, reichen aber nicht aus, um eine Sammlung zum Leben zu erwecken.

\section{Das Be-greifen von Mathematik und Informatik}

Die wichtigste Säule der Sammlung ist das Modellseminar, das im Schnitt alle drei Semester mit teils variierenden 


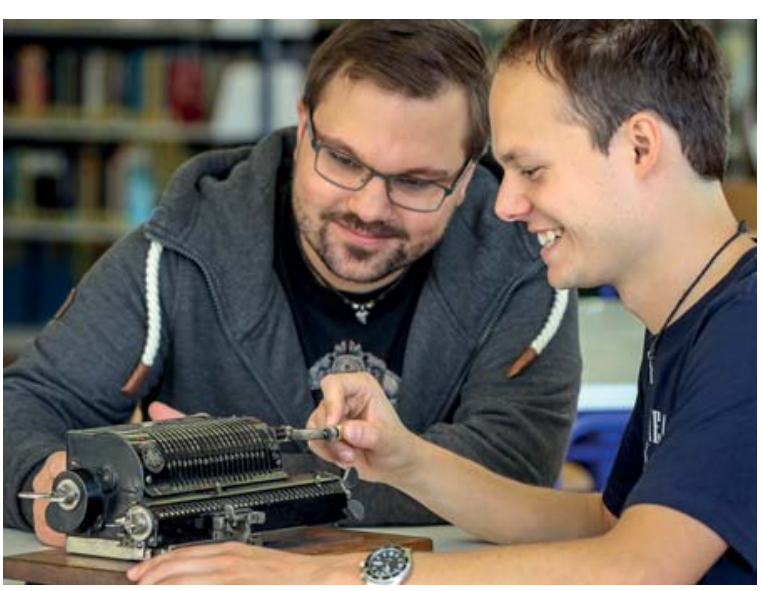

Brunsviga Sprossenradmaschine (Modell „Trinks Triplex“) von 1917 (Foto: Thorsten Richter)

Schwerpunkten für alle Studierenden des Fachbereichs angeboten wird. Ziel ist es, die Studierenden mit den Exponaten der Sammlung vertraut zu machen und die Schätze, die darin schlummern, zu entdecken. Jede Gruppe sucht sich ein Modell oder Exponat aus der Sammlung aus. Dann ist Fantasie gefragt: Jede Gruppe überlegt sich, wie man das Exponat und die fachlichen Sachverhalte, die es erläutert, den Kommilitonen näherbringen kann. Erlaubt ist alles, nur nicht 90 Minuten ohne Pause zu reden!

Typischerweise gliedern sich die Präsentationen in einen Vortrags- und einen Praxisteil: Nach einer Einführung in das Thema haben die Seminarteilnehmer die Gelegenheit, eines oder mehrere Modelle/Geräte selber zu erkunden, etwa durch Aufgaben, die in Kleingruppen an mehreren Stationen bearbeitet werden sollten. Oft basteln die Studierenden hierfür noch zusätzliche Materialien, die sehr originell sein können: etwa ein Stapel handgelochter Karteikarten auf Holzspießen, was den Sortiermechanismus eines Lochkartenrechners illustrieren soll, oder das selbstgebaute, murmelbetriebene Galtonbrett im Format I $\mathrm{m} \times 1,50 \mathrm{~m}$.

Auch die Form der Ausarbeitung ist frei wählbar. Es kann zum Beispiel ein Poster angefertigt oder ein kurzer Film gedreht werden, der auf die Homepage der Sammlung eingestellt wird, oder eine Gebrauchsanleitung für das Modell geschrieben werden, welche die Dozenten zusammen mit dem Modell ausleihen können, um es in ihren Lehrveranstaltungen zu verwenden.

Ziel ist, dass möglichst viele Dozenten einzelne Modelle in ihre normalen Lehrveranstaltungen integrieren. Viele Neuanschaffungen der letzten Jahre gehen auf konkrete Anregungen zurück, ob zur Illustration einzelner Sachverhalte geeignete Exponate zur Verfügung stehen oder besorgt werden können. Typische Lehrveranstaltungen, in denen Modelle derzeit Verwendung finden, sind:
I. Lineare Algebra (Kegelschnitte, Quadriken, ...)

2. Analysis (Rechenschieber, Integriergeräte, Flächen mit Sattel- und Wendepunkten, Cavalieri-Prinzip, ...)

3. Elementargeometrie (Körper, Polyeder, PolydronBaukasten, Pantograf, ...) und Maße und Funktionen im Mathematikunterricht (z. B. Traktograf - Eigenbau eines Gerätes, das eine Traktrix zeichnet, Pyramiden im Würfel, ...)

4. Differentialgeometrie (Spirograf, Flächen konstanter Krümmung, Gleichdick, ...),

5. Algebraische Geometrie (algebraische Flächen) und Topologie (Fraktale, Klein'sche Flasche, Möbiusband, Wurmloch, ...)

Für einige Lehrveranstaltung wurde jeweils eine Kiste angelegt, in der leichte und robuste Modelle mit Kurzbeschreibung griffbereit untergebracht sind; der Dozent nimmt dann einfach die Kiste mit in die Veranstaltung und bringt sie anschließend zurück. Größere Modelle werden separat verpackt und zur Ausleihe mitgenommen. Hier ist es von Vorteil, wenn man von einem Exponat mehrere Exemplare hat: Der Breithaupt-Pantograf von 1866 verlässt seine Vitrine natürlich nur noch selten, für Unterrichtszwecke stehen mehrere robuste Pantografen unterschiedlicher Größe zur Verfügung.

Eine ganz eigene Herausforderung ist die Erstellung eines digitalen Katalogs. Für unsere Sammlung haben wir uns entschieden, das vom Hermann von Helmholtz-Zentrum für Kulturtechnik (Berlin) angebotene Datenbankformat zu verwenden: Es ist fachlich sehr durchdacht, die Homepage zu Universitätssammlungen ist weithin bekannt, und die Funktionalität der Datenbank wird stetig weiterentwickelt. Zwar können damit bisher nur Modelle, nicht aber Messinstrumente oder Rechenmaschinen erfasst werden, aber da die systematische Katalogisierung ohnehin Jahre braucht, ist das kein wirklicher Makel. ${ }^{2}$ Langfristig bietet ein digitaler Katalog den unschlagbaren Vorteil, dass er den Nutzern der Sammlung einen guten Überblick über den Bestand liefert, weswegen kein Weg daran vorbeiführt.

\section{Und darüber hinaus?}

Mitunter ist es wünschenswert, Mathematik innerhalb eines anderen Ramens erfahrbar zu machen. Diese Funktion wird von der sogenannten Mathematischen Stadtführung erfüllt. Bei einem gemütlichen Spaziergang durch Marburg wird dabei die Stadt mit den Augen eines Mathematikers erkundet - die Botschaft lautet, dass Mathematik überall steckt, wenn man genau hinschaut. Von den Symmetrien in den Fenstern der Elisabethkirche bis zur Steigung des Weges zum Landgrafenschloss werden an verschiedenen Stationen unterschiedliche Themen behandelt. Alltägliche Dinge wie Pflastersteine, Auto-Logos oder Hydrantenschilder erscheinen in neuem Licht. 

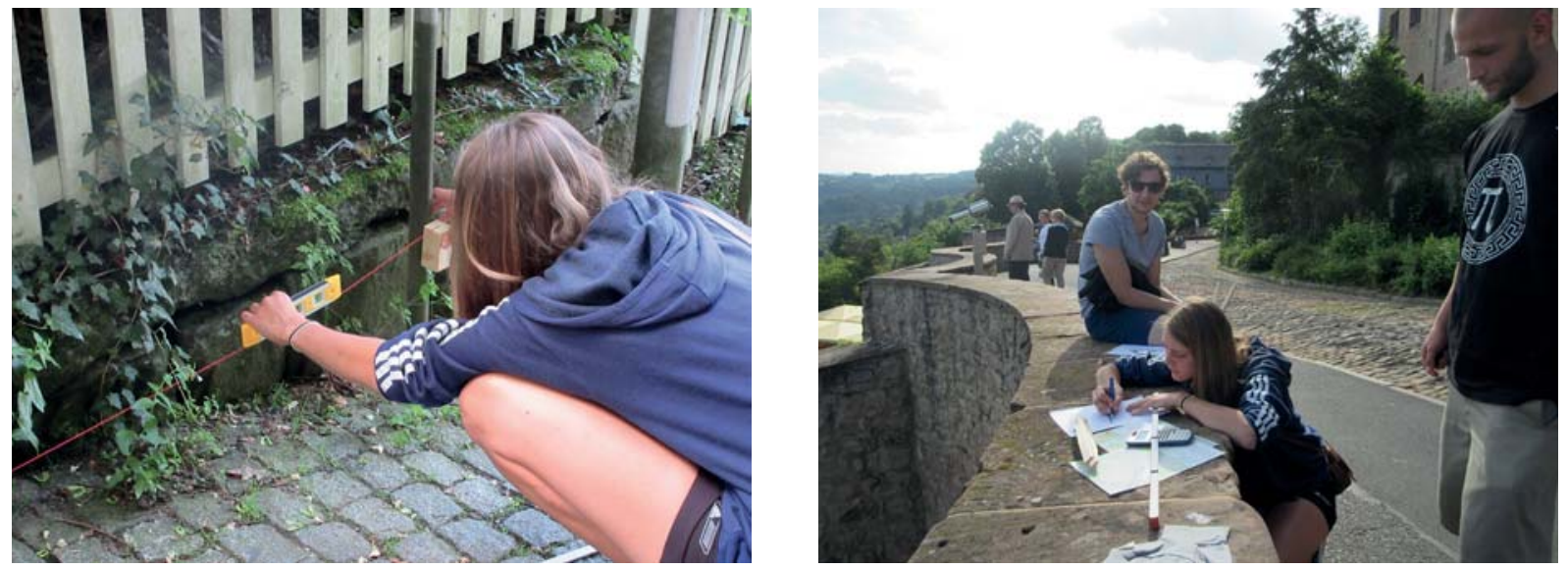

Auf der Mathematischen Stadtführung, durchgeführt im Rahmen der „MINT Summer School“ (Fotos: Korbinian Kesten)

Die Stadtführung wurde 2009 von den Studierenden des Lehramts Mathematik im Rahmen ihrer fachdidaktischen Ausbildung unter der Anleitung von Thomas Bauer konzipiert und wird seitdem stetig weiterentwickelt; seit 2014 wird sie unter dem Dach der Modellsammlung angeboten. Die erste Durchführung wurde von der Deutschen Telekom Stiftung im Rahmen des Projektes „Mathematik Vernetzen“ unterstützt. Die Mathematische Stadtführung richtet sich vorrangig an Schüler und ein mathematisch interessiertes allgemeines Publikum; darüber hinaus bietet sie den Studierenden des Lehramts eine gute Gelegenheit, während des Studiums einen ,außerschulischen Lernort" zu erkunden.

\section{Und wenn man keine eigene Sammlung hat?}

... dann gründet man eine! Beim Neuanfang 2008 war die erste Einschätzung, dass sich aus den wenigen Stücken mit etwas Glück eine Vitrine füllen lassen würde. So mancher fragte mich ungläubig: Von welcher Sammlung reden Sie eigentlich? Durch Dachbodenfunde, Dauerleihgaben und Schenkungen wuchs die Sammlung schnell an; ein Artikel im Unijournal war hier sehr hilfreich.

Es ist ratsam, sich am Anfang weniger auf Vitrinen, sondern mehr auf Modelle für die Lehre zu konzentrieren. Schulausstatter und Museumsshops bieten viel Brauchbares an, bei Christoph Pöppe $^{3}$ gibt es Kartonbausätze für Körper und bei Oliver Labs ${ }^{4}$ kann man günstig wunderbare Modelle kaufen, die mit dem 3D-Plotter gedruckt werden. Mit einem Startkapital deutlich unter 5000 Euro, einem Raum und einer studentischen Hilfskraft kann man bereits eine sehr ordentliche Sammlung gründen und sukzessive ergänzen. Denn frei nach Goethe gilt: So eine Sammlung wird eigentlich nie fertig, man muss sie für fertig erklären, wenn man nach Zeit und Umständen das mögliche getan hat ... und wenn man wieder mehr Zeit hat, macht man einfach weiter.
Anmerkungen

I. Homepage der Sammlung: www.mathematik.uni-marburg. de/fb $12 /$ modellsammlung

2. Den bisherigen Stand kann man unter folgender Adresse einsehen: http://www.universitaetssammlungen.de/modelle/ suche/sam/I 203

3. http://www.poeppe-online.de/

4. http://blog.mo-labs.com/

\section{Literatur}

Ilka Agricola, Die Mathematische Modellsammlung, in: Schätze der Wissenschaft - Die Sammlungen, Museen und Archive der PhilippsUniversität Marburg. Herausgegeben von Christoph Otterbeck und Joachim Schachtner, Jonas Verlag, Marburg (20I4).

Ilka Agricola, Wolfgang Gromes, Webseiten zur Geschichte des Fachbereichs Mathematik und Informatik, http://www.mathematik. uni-marburg.de/fb I/2/historie

Karl-Bernhard Gundlach, 100 Jahre Mathematisches Seminar - Ein Rückblick auf die Entwicklung der Mathematik in Marburg, erweiterte Fassung eines Vortrags zur Feier des hundertjährigen Bestehens des Mathematischen Seminars der Universität Marburg, 1985, erhältlich unter http://www.uni-marburg.de/fb I/historie/ 100-jahre-mat-sem

Prof. Dr. Ilka Agricola, FB I2, Mathematik und Informatik, PhilippsUniversität Marburg, Hans-Meerwein-Straße/Campus Lahnberge, 35032 Marburg

agricola@Mathematik.Uni-Marburg.de

Ilka Agricola ist seit 2008 Professorin am Fachbereich Mathematik und Informatik der PhilippsUniversität Marburg und seitdem wissenschaftliche Leiterin der Mathematischen Modellsammlung. Wenn sie nicht gerade während der Arbeitszeit mit Modellen spielt, forscht sie auf dem Gebiet der Differentialgeometrie und SpinGeometrie. Zusammen mit Thomas Friedrich veröffentlichte sie Lehrbücher zur Elementargeometrie und Vektoranalysis. 